\title{
SIMULATION AS A DECISION SUPPORT TOOL FOR AIRPORT PLANNING: RIGA INTERNATIONAL AIRPORT CASE STUDY
}

\author{
Mihails SAVRASOVS, Irina YATSKIV (JACKIVA)”, Jurijs TOLUJEVS, Ilya JACKSON* \\ Faculty of Engineering, Transport and Telecommunication Institute, Riga, Latvia
}

Submitted 25 January 2021; resubmitted 26 May 2021; accepted 8 June 2021

\begin{abstract}
This research considers the aspects of decision-making according to the airport activities. The decision about airport planning and management should be comprehensive and operative and of course, the assessment of alternative decisions is necessary. The purpose of this research is to highlight the role of simulation modelling at the stage of airport development. The authors present the methodology of a model-driven decision-making approach and then describe 2 cases of using simulation for Riga International Airport (RIX) development. The 1st case study is used for analysis possibility of the development of the airport's surrounding territory. The planned massive development of RIX and the surrounding area requires detailed analysis for increasing its positive impact on regional and national business economics, social aspects, business and the environment. The 2nd case supports decision-making for the needs of the terminal reconstruction, presents a helpful tool for visualization of the tendencies in the future, and allows the analysis of the different infrastructure layouts. Both cases give the possibility to predict the situation and evaluate the service provided for passengers (travellers) of the airport. Simulation modelling allows to study complex system - airport and evaluate direct and indirect impacts of planned reconstruction.
\end{abstract}

Keywords: airport, decision, levels, models, case study, traffic volume, passenger.

\author{
Notations \\ CBA - cost-benefit analysis; \\ DM - decision-maker; \\ DSS - decision support system; \\ LoS - level of service; \\ MoE - measures of effectiveness; \\ RIX - Riga International Airport; \\ TAAM - total airspace and airport modeller; \\ TIA - traffic impact analysis; \\ WSC - winter simulation conference.
}

\section{Introduction}

Solving managerial tasks in complex systems lies in the domain of designing the DSS, and the primary attention is paid to the process, not to the result of the decision or the essence of the problem. Decision-making is a conscious choice between the existing variants or alternatives of the direction of actions, which reduce the break between the present and the future desired state of the object of management (Yatskiv (Jackiva) et al. 2016).

The decision on the planning (reconstruction) of transport infrastructure is one of the most important and needs thorough analysis and prior consideration. Often, in making project decisions, there is a lack of sufficient information. Therefore, the DM has to deal with uncertainty and risk. In connection with this, the problem of decisionmaking support in choosing a project decision on building new and reconstructing existing transport infrastructure objects takes on special significance and requires improvement of the methodology of its decision. In this research, attention is paid to issues that have been solved for DSS development, which has to support the process of complex system planning and development.

In the research, the aspects of decision-making according to the airport activities were considered. The decision about airport planning and management should be comprehensive and operative and of course, the assessment of alternative decisions is necessary. This research aims to highlight the role of simulation modelling at the stage of airport development.

The paper is organised as follows:

"» section 1 presents a brief overview of related works;

"» section 2 introduces our methodology of modeldriven decision support; 
"» section 3 presents the research object - RIX;

"» sections 4 and 5 describe the case studies, obtained results and related discussion;

"» last section summarizes the conclusions.

\section{Related works}

This section sheds light on prominent applications of simulation models for the enhancement of decision-making at various airport operations. The literature review presented in this section is mainly based on papers published at proceedings of the WSC, the premier international forum for disseminating recent advances and breakthroughs in the field of applied simulation modelling.

In recent study, López et al. (2019) presented a discrete event simulation model integrated into the transactional flight database. A series of simulation experiments and consideration of various alterative scenarios allowed one to identify the optimal bus fleet size. Aircraft turnaround operations including passenger boarding, disembarking and re-fuelling were taken into account by a robust tactical scheme for efficient resource allocation in cases of operational disruptions. The scheme is based on a combination of ad-hoc heuristics with an agent-based simulation model (Tomasella et al. 2019). A general approach for modelling airport operations was presented by Scala et al. (2019). The distinguishing feature of the proposed approach is that the resolution level for the different elements is similar. It is suggested that practitioners can use the framework for simulating complex systems including airspace-airside operations and multi-airport systems. The framework is tested on a real-world case study.

The problem of developing robust daily schedules for maintenance teams and vehicle routing recently attracted attention of Gök et al. (2020). The authors proposed a robust approach for optimal allocation time windows and planning working shifts. The core methodology combines simulation modelling and mixed integer programming. The obtained numerical results demonstrated applicability of the developed approach as a decision support mechanism at airports.

Scala et al. (2018) presented an innovative approach for solving complex capacity-related problems in aviation. A sliding window framework that incorporates discrete event simulation model. The authors conclude with the statement that the framework can be applied to solve capacity planning problems in aviation industry. The approach was validated based on the Paris Charles de Gaulle Airport (France). A comprehensive simulation environment is proposed to optimize aircraft boarding taking into consideration the influence of infrastructural changes and aircraft design (Schultz 2017). Tomasella et al. (2017) developed a simulation-based toolkit for improving the existing baggage handling system. Vitor et al. (2016) developed a simulation-driven approach to improve checkin at Congonhas Airport in Sao Paulo (Brazil). The study considered 2 major airlines, which account for $88 \%$ of the local market share.
Another study illustrated how simulation could be used to analyse critical aircraft turnaround processes at airports (San Antonio et al. 2017). A series of numerical experiments considering the Boeing 737-800 aircraft was presented. Alodhaibi et al. (2020) developed a simulationbased DSS to allocate physical resources within airport terminals. The primary purpose of the framework is to determine where additional resources should be placed in order to minimise passenger waiting times. The recent paper proposed the use of simulation model as a testbed for solutions in the field of centralized traffic control at airports (Saifutdinov et al. 2020). A distinguishing feature of the model is the ability to describe and reproduce specific scenarios related to critical situations in the transport network that require the involvement of a centralized control system.

\section{Methodology of using simulation models in decision-making}

Considering the complex character of airport planning and management, the DM needs a methodology for supporting the decision-making process. Several approaches to decision-making were distinguished (Ortúzar, Willumsen 2011): the one based on development plan, the theory of regulatory decisions (rational system approach), the behavioural decision theory, the group decision-making, the adaptive decision-making, and making decisions based on a hybrid approach.

The decision-making process involves many different elements but it always has elements such as problem, goals, alternatives and decisions - choice of alternatives. There are DSS widely used as a means for information support of the decision-making in the field of transport system planning, development and control. Ginzberg and Stohr (1982) define DSS as “...a computer-based information system used by DMs to support their decision-making activities in the situations where it is not possible or not desirable to have an automated system perform the entire decision process..." The DSS for transport planning helps the DM to modify the information as required to define alternatives before he starts the decision process. More common questions that answered based on modelling can be formulated as:

"» does the infrastructure provide enough capacity?;

"» how many resources (staff, check-in points, security control places, equipment) are required?;

"'» is the layout of the infrastructure stable, i.e., insusceptible to failures and changes?;

"»" are there enough parking spaces and what are the best locations for parking and preparation?;

"» etc.

According to Cascetta et al. (2015), the combination of various factors, decisions in transportation decisionmaking can be grouped into 4 classes:

"» long-term decisions by public bodies incl. transportation infrastructure projects;

"» long-term decisions by private companies incl. project financing and fleet composition; 
"» medium/short-term decisions by public bodies incl. traffic regulation, demand management schemes, intelligent transportation systems;

"» medium/short-term decisions by private companies incl. operation of transportation services.

Table 1 presents characteristics of decision classes depended on time horizons and involved stakeholders.

DSS may be classified as expert-based, model-based, multi-agent systems and the combined type (Yurshevich 2013). In this research, the model-based approach is considered. Using models for decision support provides the opportunities for the development of credible solutions: the models allow one to evaluate the feasibility of scenario-based solutions of the existing problems, to predict the future state of the system and possible consequences of putting the decisions into execution, to help in finding such scenario that would achieve the goal under given constraints. Therefore, an evaluation of alternative solutions is easy to perform on the models. There are dedicated DSSs special and general types, allowing one to manage the passenger or airport vehicle traffic at different levels of decision-making. The implementation of the model-driven decision-making approach requires the use of modern technology, modelling, various kinds of data and information. The scales of models and data strongly depend on different decision-making levels: strategic, tactical or operational (Figure 1).

There are 3 approaches to transport modelling: macroscopic, mesoscopic and microscopic, which could be linked to model-based decision-making levels. Different levels of simulation modelling approaches are used for different decision-making levels:

"» macro-level (high abstraction level without details) for strategic level;

"») meso-level (medium abstraction level) for tactical level. At the tactical level, specialists deal with the reconstruction of the existing fragments of systems and developing the new ones, taking into account

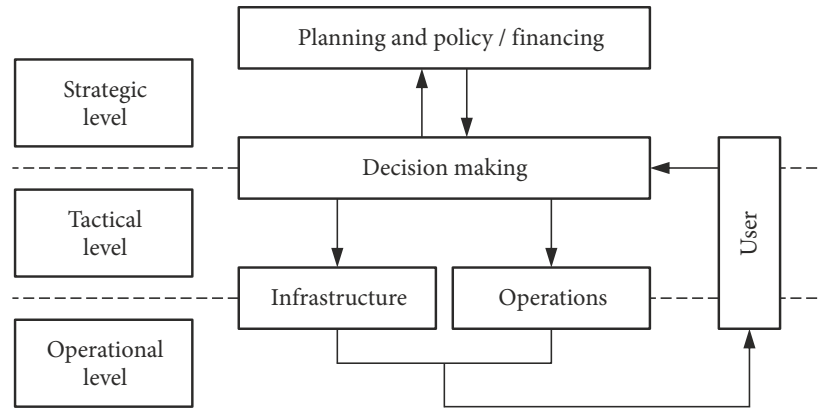

Figure 1. Decision-making framework (CLOSER 2012)

the applicable strategic plan for the development of systems in the long-term. Usually, at the tactical level, the objectives are set for the medium term (up to 5 years);

"» micro-level (low abstraction level without full details) for operational level. At the operational level, the local problems existing in specific fragments of the systems are solved at the micro-level.

Figure 2 illustrates the generalized framework for model-based decision-making.

In this research the mixed type of decision-making has been selected which includes the following steps:

"» the formulation of the problem;

"» the system monitoring and data collection;

"» the construction of analytical and simulation models, and their subsequent calibration;

"»" generation of solutions of current problems, and forecasting the system parameter values being of interest;

"»" validation of models and solutions;

"» assessment of the possible consequences of the implementation of certain decisions, and selecting the best ones;

"» putting solutions into execution with the subsequent monitoring of the system to identify potential problems.

Table 1. The combination of various factors, decisions in transportation decision-making (Cascetta et al. 2015)

\begin{tabular}{|c|c|c|}
\hline Decisions & By public bodies & By private companies \\
\hline Long-term & $\begin{array}{l}\text { "'» multiple DMs and strong impacts on contrasting stake- } \\
\text { holder interests; } \\
\text { "»" complex financial procedures; } \\
\text { "» high level of uncertainty and relevant impact/context vari- } \\
\text { ables; } \\
\text { "»" strong potential interactions with other systems (e.g., land } \\
\text { use, economy, etc.); } \\
\text { "»" zero to low levels of reversibility for implemented decisions }\end{array}$ & $\begin{array}{l}\text { "» decisions with moderate to high impacts on stake- } \\
\text { holders including public bodies and financial insti- } \\
\text { tutions; } \\
\text { "» high level of uncertainty on relevant impact/context } \\
\text { variables; } \\
\text { "» moderate interactions with other systems (e.g., con- } \\
\text { struction industry); } \\
\text { "» low levels of reversibility for implemented decisions }\end{array}$ \\
\hline $\begin{array}{l}\text { Medium/ } \\
\text { short-term }\end{array}$ & $\begin{array}{l}\text { "» identifiable DMs with clear responsibility, wide range of in- } \\
\text { teractions with stakeholders (from very high to none); } \\
\text { "» systematic decision procedures in the presence of a close } \\
\text { set of alternative project options, with quantitative design } \\
\text { variables; } \\
\text { "» low level of uncertainty on relevant impact/context vari- } \\
\text { ables; } \\
\text { "» low interactions with other systems; } \\
\text { "» high level of reversibility }\end{array}$ & $\begin{array}{l}\text { "» identifiable DMs with clear responsibility, low in- } \\
\text { teractions with stakeholders (e.g., workforce); } \\
\text { "» relatively straightforward decision procedures; } \\
\text { "» low level of uncertainty on relevant impact/context } \\
\text { variables; } \\
\text { "» low interactions with other systems; } \\
\text { "» high level of reversibility }\end{array}$ \\
\hline
\end{tabular}




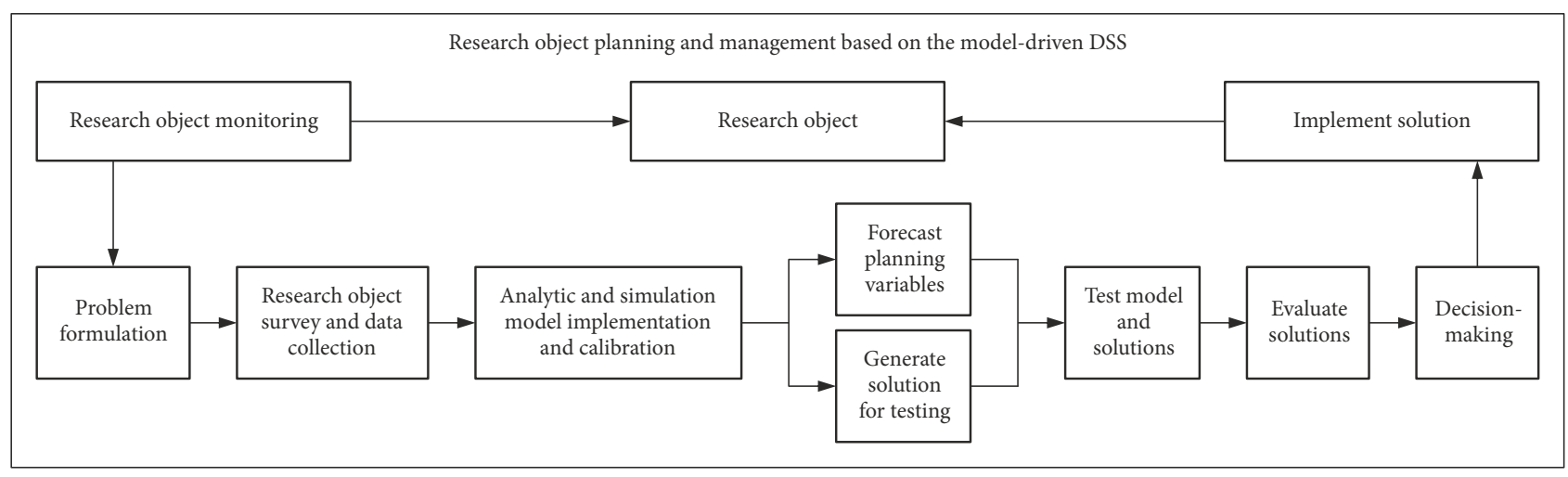

Figure 2. Framework of model-based decision-making

The adequacy of decisions depends on the validity of simulation results and model calibration is one of the most critical steps of the model creation process. Model calibration can be performed based on aggregated or disaggregated data. The use of disaggregated data allows one to perform a more precise calibration, with car following trajectories and vehicle speed data being frequently used. Measured characteristics are grouped based on the time and place of measurement. Disaggregated data is collected using special-purpose equipment; it is an expensive process that cannot always be afforded. It also requires continuous monitoring of system state, data collection, processing and analysis, playback scenarios of development and estimation of possible solutions. The implementation of these models depends on the selected modelling tool and the inbuilt analytical models. These analytical models are the "heart" of software, and the quality of their implementation directly influences the accuracy of simulation results. Several general purpose simulations and special (for airport process) simulation software products can be used for scenario modelling. However, the choice of a specific one also depends on simulated objects (process) and the level of the decision. In this research, we only consider the processes that include the different kinds of traffic: aircraft traffic in the airport, airside and apron traffic, passenger flow traffic, baggage flow handling and traffic at the airport network.

Usually the development of simulation models requires using of specific software. In a professional context, the following software packages are more famous: airport simulation software CAST by the Airport Research Center (ARC 2021); Simmod US (ATAC 2018); TAAM (Jeppesen 2015, 2021), etc. Usually, this software is costly and demands advanced specialised users. General purpose simulation software includes various tools: Simio (Simio LLC 2016), FlexSim (FlexSim Software Products, Inc., US); Enterprise Dynamics (INCONTROL Simulation Solutions, the Netherlands); AnyLogic (AnyLogic North America LLC, US; AnyLogic Company, Russia). In this research, the PTV VISSIM (PTV 2005) and AnyLogic (https://www.anylogic.com) simulation software have been used.

\section{Airport RIX as a research object}

The RIX is a fast-growing Northern European transportation hub and is leading across Baltic States (Latvia, Lithuania, and Estonia) by the number of passengers, connecting the Baltic States with European business centres and popular holiday destinations. The RIX is geographically located in $10 \mathrm{~km}$ west of Riga but still can be considered as the transportation hub of the city of Riga. The RIX serves nearly half of all Baltic States passengers and offers the broadest range of direct destinations compared to other Baltic States airports. RIX provides services for both aviation and non-aviation. It serves both national and international airlines and become one of the few European airports to facilitate both full and low-cost airlines (RIX 2021).

RIX is the largest international aviation company in the Baltic States with a market share of $45 \%$ and the central air traffic center in this region offering regular passenger, cargo and postal delivery to the cities of Europe and the world. It attends both national and international airlines, becoming one of the few European airports that attend both full service and low-cost airlines (RIX 2021). In 2017, RIX welcomed $6.1 \mathrm{mln}$ passengers. According to the ranking of Airports Council International (https://aci.aero), RIX has officially joined the ranks of medium-sized airports and internationally will not be treated as a small airport anymore. The airport has completed its phase 5 terminal expansion project, which will allow accommodating 7 to $10 \mathrm{mln}$ passengers annually, cater to large aircraft, and develop long-haul flights (BRG 2017). In the last decade, the Latvian government has made massive investments in the airport infrastructure, and additional projects are implemented. RIX is State Joint-Stock Company and almost all of the passenger flights $99 \%$ are performed from the Riga (MoT 2006).

The continuous growth of passengers (Figure 3 ) pushes the development of airport infrastructure, but at the same time surrounding transport infrastructure requires a significant update to meet the requirements of service quality, safety, and capacity. In the context of the pandemic-related aviation industry crisis, the operational performance data show that in 2020-2021 the number of passengers 


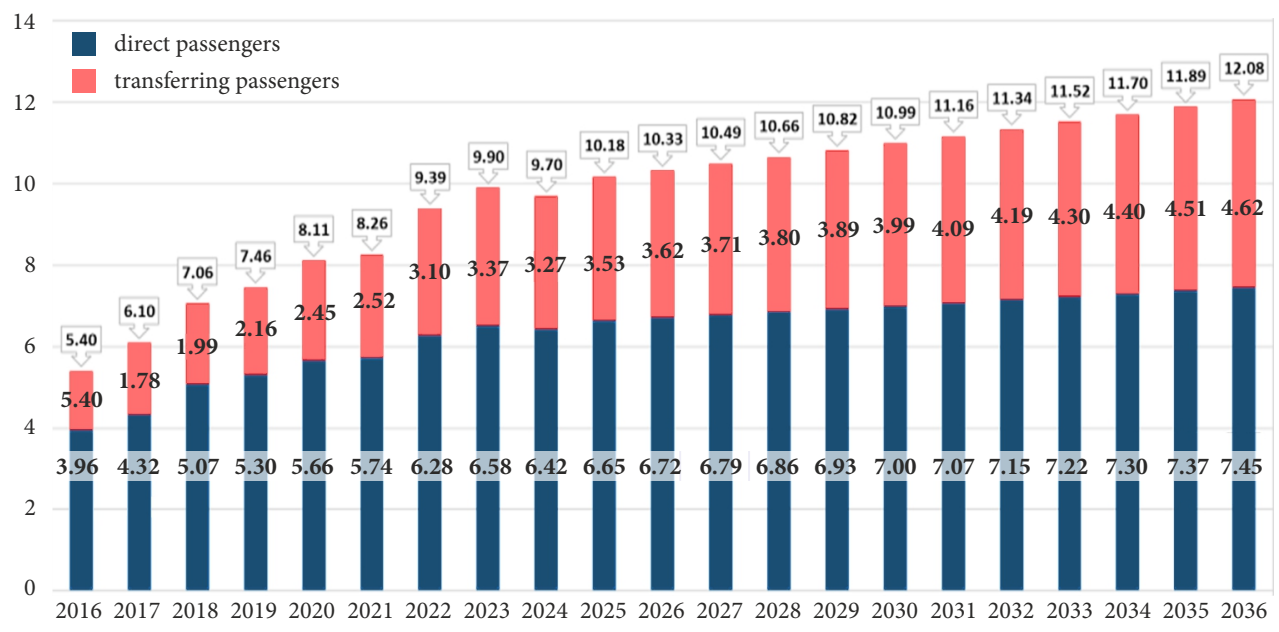

Figure 3. Passenger flow forecast (RIX 2021)

served at RIX dropped significantly and the planned traffic growth will be achieved with substantial delay. Despite this fact, the development of the airport has not been stopped since already with the previous traffic volumes (2018-2019), the airport experienced problems due to insufficient areas (capacity). As a member of the European Union Aviation Safety Agency (EASA, https://www. easa.europa.eu) Aviation Industry Charter for COVID-19 (https://www.easa.europa.eu/aviation-industry-chartercovid-19), RIX participates in the development of the highest standards of epidemiological safety. SKYTRAX Company (https://skytraxratings.com) has awarded RIX a 4-star rating for its implemented COVID-19 safety measures. The airport hopes to restore the volume of transfers as soon as possible.

The proposed approach to use simulation modelling as a decision-making support tool for airport planning is implemented in different projects developed in SimLab (Laboratory of Applied Modelling, Transport and Telecommunication Institute, Riga, Latvia) (TTI 2021). 2 case studies described below give possibilities to perform an impact assessment (case 1 - traffic flow; case 2 - passenger flow), incorporating factors that influence the reliability of airport services and support to decision-making in the development of facilities.

\section{Case study: RIX surrounding transport network development scenario evaluation}

\subsection{Simulation set-up}

The development of the RIX transport infrastructure is planned by the development stages and is reflected in the Figure 4.

The main goal of this project was to evaluate the different development scenarios and complete a TIA. The decision-making approach based on simulation modelling is presented in Figure 5.

Traffic flow microscopic simulation has been selected as an appropriate approach to complete analysis. The de- velopment of the model has been completed using PTV VISSIM simulation software (PTV 2005). Figure 6 demonstrates the network model coded in PTV VISSIM simulation software. In total network consist of more than 300 link and connector objects, 14 signal heads, 6 public transport stops and 2 public transport lines, more than 30 parking lots, more than 50 conflict areas, 44 speed objects.

Microscopic model development requires detailed data about the transport network, traffic lights, traffic and passenger flows, behaviour patterns of traffic and passenger flows. Network topology and traffic light data has been received from the municipality. The data about the structure, intensity, and behaviour has been completed during peak hours (11:00-13:00) to set up the system's current state. The example of the collected data is represented in Figure 7.

\subsection{Model validation and calibration}

The current state model has been validated using 3 approaches:

"» based on animation: the group of experts (managerial staff of airport) observed the animation produced by the model (Figure 8); the developed model has been approved as credible by experts;

"» using the GEH index: In all cases, GEH index values were less than 5; this confirms the model's validity; Table 2 demonstrates the numerical results of the GEH index evaluation;

"» applying the NAÏVE approach: high value or $R^{2}=0.98$ confirms the validity of the model (Figure 9).

\subsection{Results of modelling}

In total 10 development scenarios have been evaluated with different reference years - 2021, 2022, 2025, 2036. Each scenario is subject to changes in traffic and passenger flow intensity and changes in the transport network. Figure 10 demonstrates the hierarchy of the scenarios. 

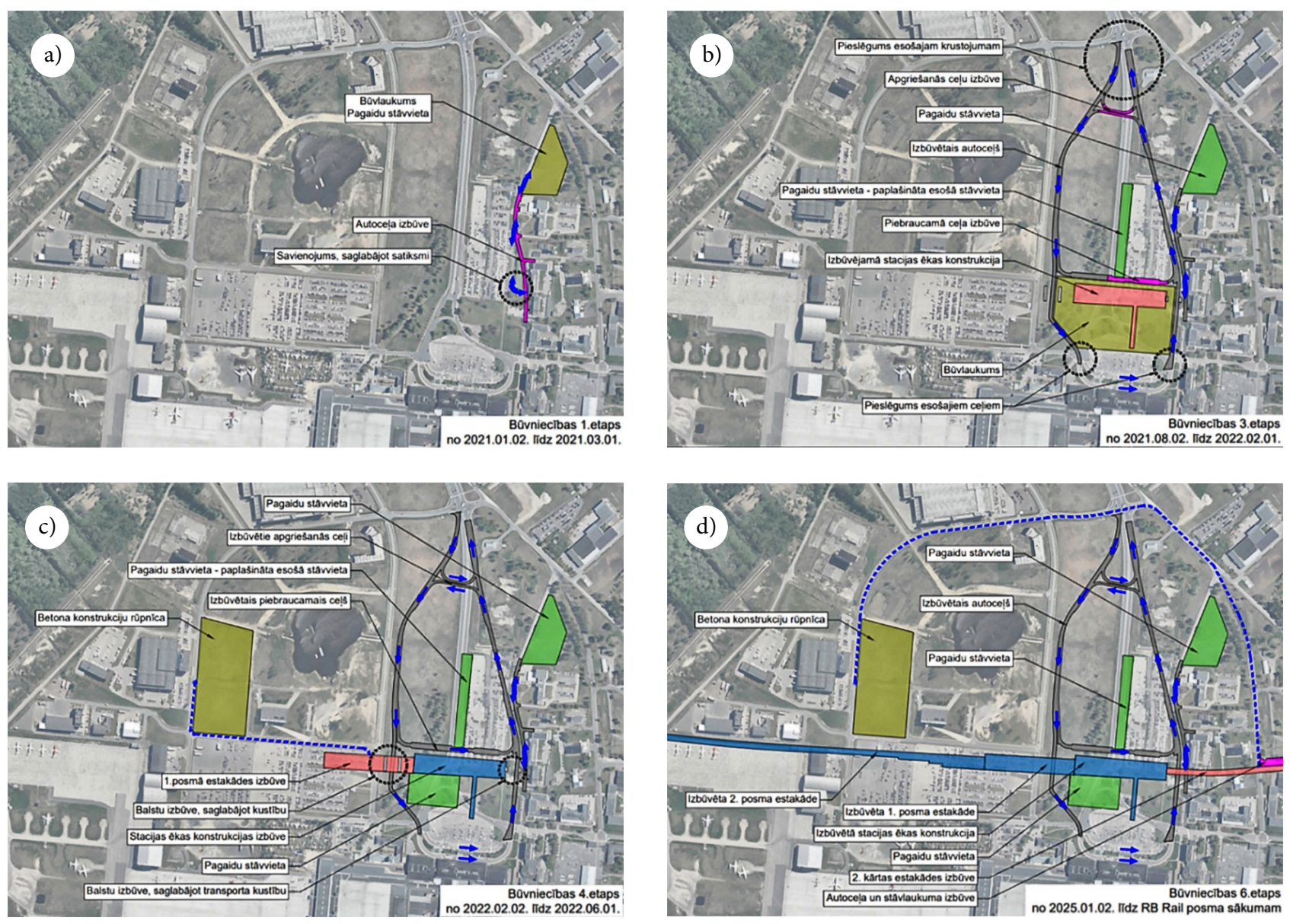

Figure 4. Stages of airport development: $\mathrm{a}-1$ st stage of development; $\mathrm{b}$ - 3rd stage of development; $\mathrm{c}-4$ th stage of development; $\mathrm{d}-6$ th stage of development

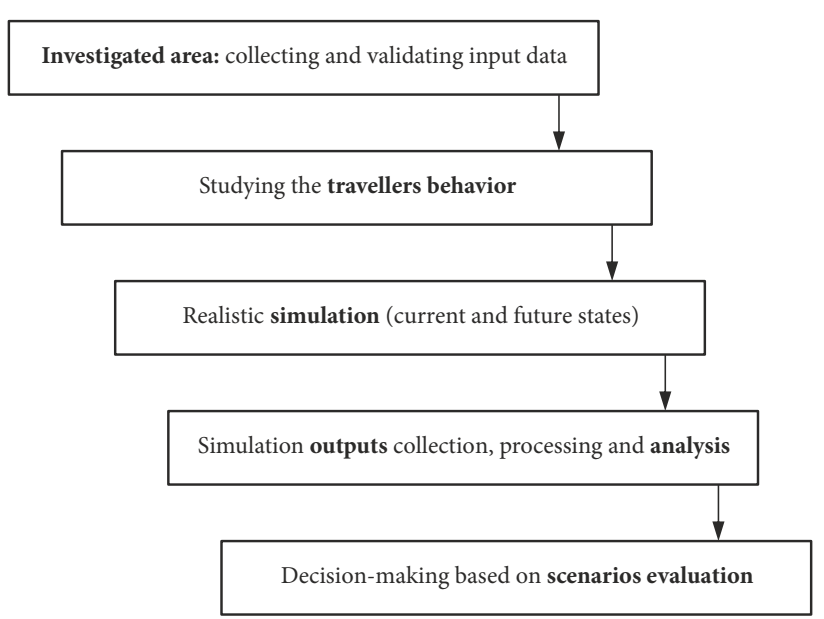

Figure 5. Model-driven decision-making approach for traffic impact assessment

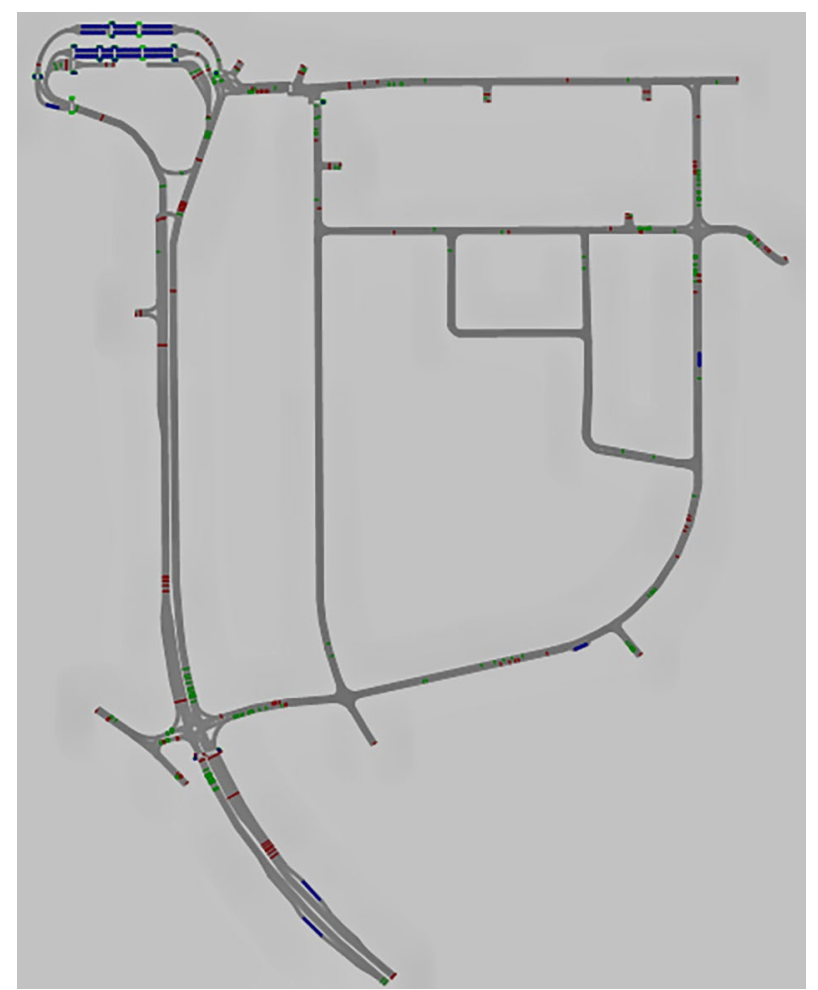

Figure 6. Network model 
a)

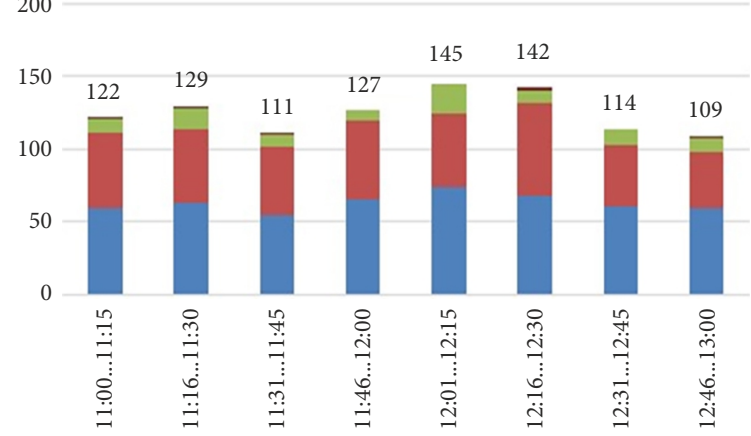

b)

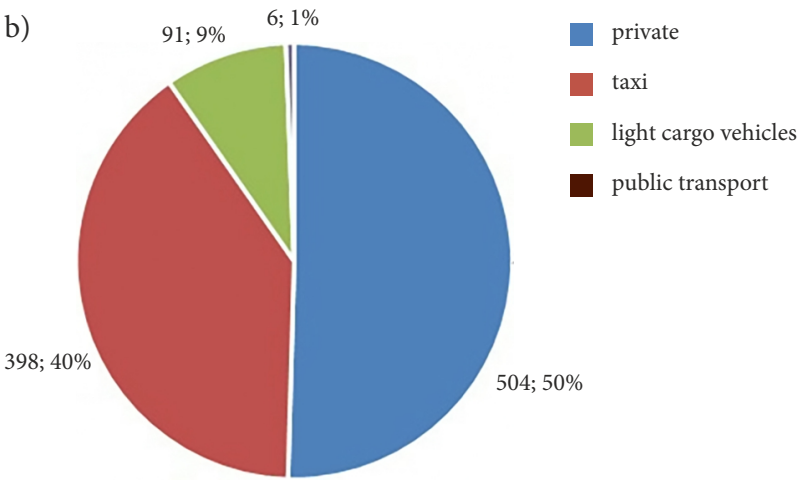

Figure 7. Example of traffic data near departure terminal: $a$ - traffic flow intensity; b - traffic flow structure

Table 2. Results of validation using GEH index

\begin{tabular}{|l|l|c|c|c|}
\hline \multicolumn{1}{|c|}{$\begin{array}{c}\text { Counting } \\
\text { point }\end{array}$} & Directions & $\begin{array}{c}\text { Simulated } \\
\text { volume }\end{array}$ & $\begin{array}{c}\text { Real } \\
\text { volume }\end{array}$ & $\begin{array}{c}\text { GEH } \\
\text { (rounded) }\end{array}$ \\
\hline \multirow{2}{*}{ RIX ramp } & to RIX & 938 & 999 & 2 \\
\cline { 2 - 5 } & from RIX & 920 & 995 & 2 \\
\hline $\begin{array}{l}\text { Crossroad } \\
\text { P133 }\end{array}$ & to RIX & 1414 & 1349 & 2 \\
\cline { 2 - 5 } & from RIX & 1411 & 1475 & 2 \\
\hline $\begin{array}{l}\text { Muzeja iela } \\
\text { (street) }\end{array}$ & to RIX & 339 & 368 & 2 \\
\cline { 2 - 5 } & from RIX & 165 & 156 & 1 \\
\hline
\end{tabular}

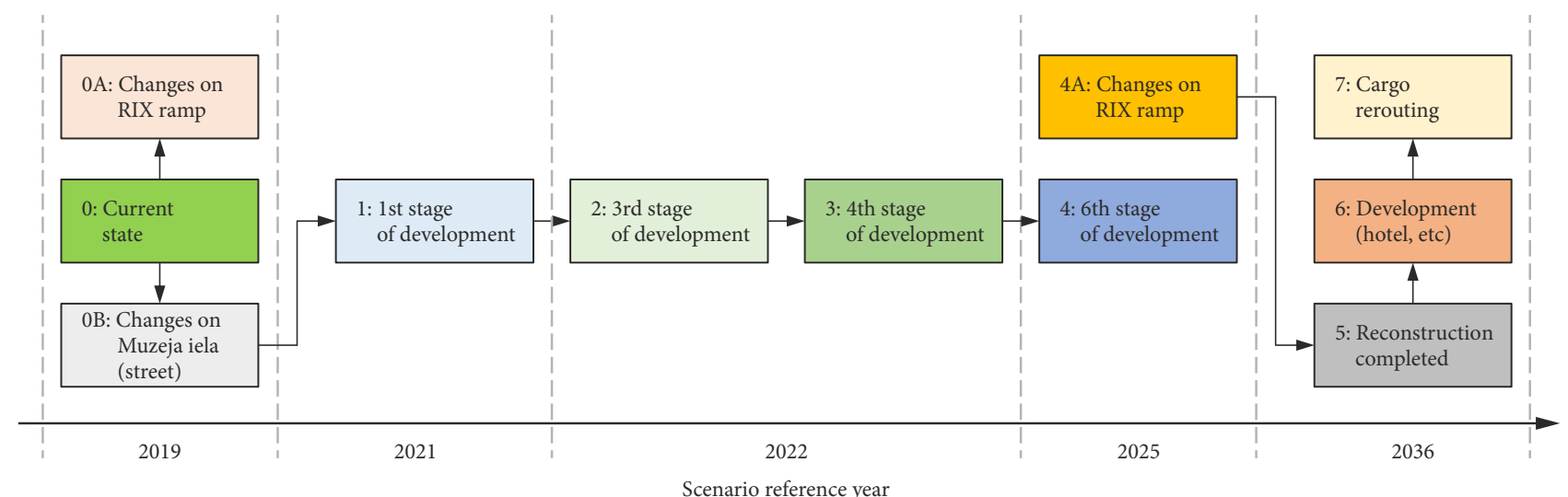

Figure 10. Scenario hierarchy

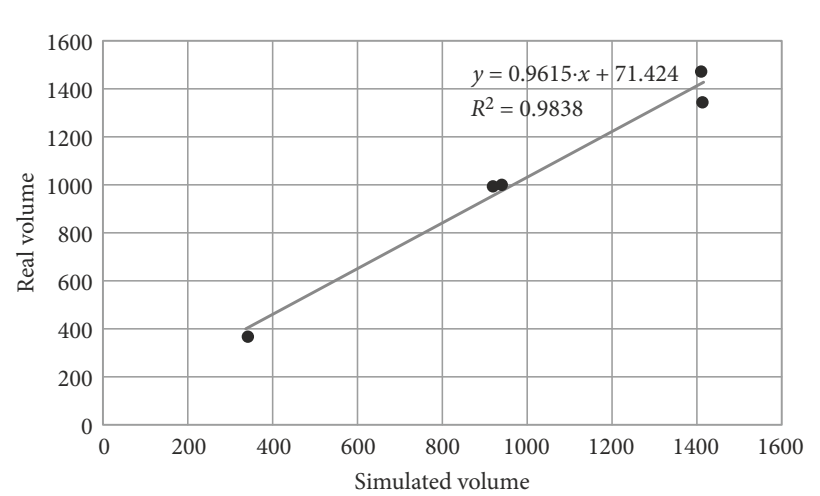

Figure 9. NAÏVE approach validation results

)

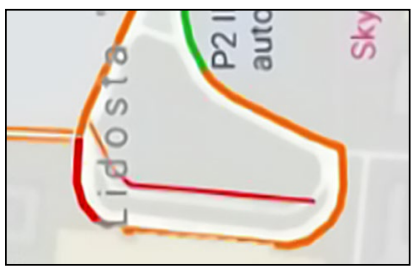

b)

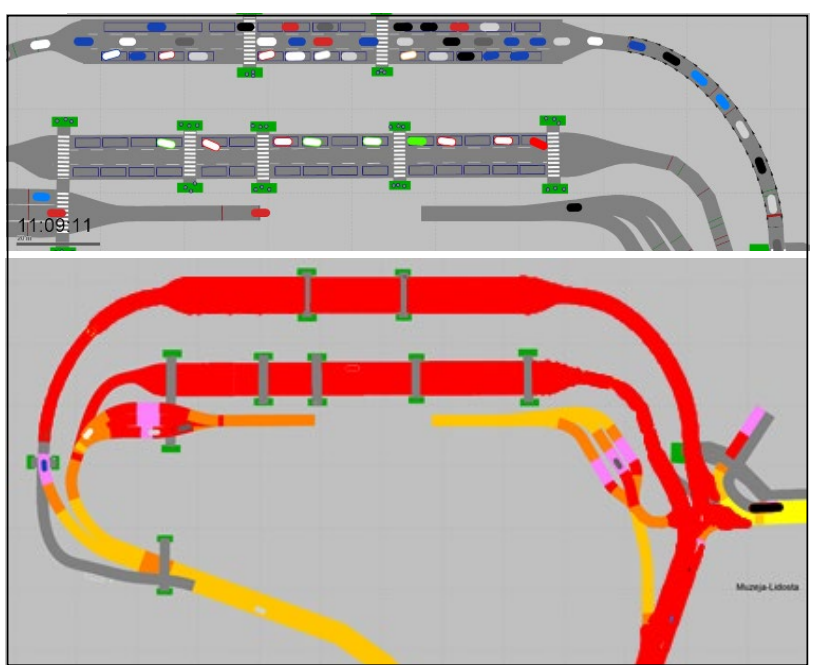

Figure 8. Calibration based on animation: a - Google Maps data; b - animated model 
Table 3. Simulation results

\begin{tabular}{|l|l|l|l|}
\hline \multicolumn{1}{|c|}{ Scenario } & \multicolumn{1}{|c|}{ Ziemel̦u iela (street) - Crossroad P133 } & \multicolumn{1}{c|}{ Rail Baltica terminal } & \multicolumn{1}{c|}{ RIX terminal } \\
\hline Scenario 4A (2025) & LoS: C & LoS: B & $\begin{array}{l}\text { LoS: C } \\
\text { Parking utilisation: } 51 \%\end{array}$ \\
\hline Scenario 5 (2036) & $\begin{array}{l}\text { LoS: C } \\
\text { Direction from city centre to RIX in average is 30 m }\end{array}$ & $\begin{array}{l}\text { LoS: C } \\
\text { Parking utilisation: } 80 \%\end{array}$ & $\begin{array}{l}\text { LoS: C } \\
\text { Parking utilisation: 70\% }\end{array}$ \\
\hline Scenario 6 (2036) & $\begin{array}{l}\text { LoS: D } \\
\text { Direction from city centre to RIX in average is 45 m }\end{array}$ & $\begin{array}{l}\text { LoS: C } \\
\text { Parking utilisation: 84\% }\end{array}$ & $\begin{array}{l}\text { LoS: C } \\
\text { Parking utilisation: } 72 \%\end{array}$ \\
\hline Scenario 7 (2036) & LoS: C & $\begin{array}{l}\text { LoS: C } \\
\text { Parking utilisation: } 84 \%\end{array}$ & $\begin{array}{l}\text { LoS: C } \\
\text { Parking utilisation: } 72 \%\end{array}$ \\
\hline
\end{tabular}

Table 4. Sensitivity analysis (scenario 5)

\begin{tabular}{|c|c|c|c|}
\hline $\begin{array}{c}\text { Grow of } \\
\text { the traffic } \\
\text { intensity [\%] }\end{array}$ & $\begin{array}{c}\text { Ziemel̦u iela (street) } \\
\text { Crossroad P133 }\end{array}$ & $\begin{array}{c}\text { Rail Baltica } \\
\text { terminal }\end{array}$ & $\begin{array}{c}\text { RIX } \\
\text { terminal }\end{array}$ \\
\hline 0 & LoS: C & LoS: C & LoS: C \\
\hline+5 & LoS: C & LoS: C & LoS: C \\
\hline+10 & LoS: D & LoS: C & LoS: C \\
\hline+20 & LoS: $\mathrm{D}$ & LoS: $\mathrm{D}$ & LoS: $\mathrm{D}$ \\
\hline+30 & LoS: $\mathrm{D}$ & LoS: $\mathrm{D}$ & LoS: $\mathrm{E}$ \\
\hline
\end{tabular}

Each scenario has been evaluated based on the MoE: average speed in network, average delay time, LoS for key intersections, travel time, queue lengths. Additionally, the capacity of the parking areas has been evaluated. To collect the output data, the following conditions have been setup: simulation time $1 \mathrm{~h} 45 \mathrm{~min}, 15 \mathrm{~min}$ - warming period (no data collection during this time), 25 replications per scenario. Due to a high number of scenarios in Table 3 we are demonstrating only the most critical scenarios results. Scenario 5 is a key one as this scenario considers that reconstruction of the terminal and surrounding transport infrastructure has been completed. Therefore, the sensitivity analysis has been completed to determine the critical level of the traffic flow growth. The sensitivity analysis results are presented in Table 4 . As could be seen from the table, up to $10 \%$ growth of the traffic volume does not influence significantly on LoS level in crossroads. As LoS D is treated as the last acceptable, it also could be concluded, that grown of intensity up to $30 \%$ changes LoS to unacceptable for RIX terminal.

Based on the received simulation results, several bottlenecks have been identified, which could influence traffic flow circulation in the future; but in general, planned changes in the transport network will ensure the sustainable operation of the transport system.

\section{Case study: RIX passenger flow simulation and analysis}

As highlighted by Chen et al. (2019), airport construction is capital intensive, and any mistake will be costly to recover and ne feasible way to test out the terminal de- sign without a high cost through simulation. As has been stated in the previous case, the number of passengers is continuously growing. Currently during peak hours, the situation with passenger flows at terminals becomes dramatic, especially in the security area. The next project aims to evaluate several vulnerability-mitigation scenarios that can potentially decrease the waiting time in queues. Besides, the capacity of the planned new terminal has been tested for expected growth in passenger traffic.

\subsection{Simulation set-up}

A simulation study has been conducted within the discrete-event simulation paradigm based on previously collected real-world data. The set of models has been developed using AnyLogic simulation software, which allows passenger flow modelling on a micro-level.

A valid discrete-event model requires valid data on the intensity of passenger flows, behaviour patterns, the topology of the terminal and many other variables. The used data included the entire day at the airport and special interval of interest: check-in starts at 8:55 and check-in ends at 14:55 (Table 5). This is the busiest period for the airport, both in terms of the number of passengers and the availability of check-in counters. The data also includes passenger service times, which vary from company to company, taking into account the passenger habits and the range of services and requirements offered by the airline. The model takes into account security control data, one line serves an average of 120 passengers per hour (this applies to both high-speed and regular lines). The facility profile is designed based on the type of activities that are available in the terminal area.

During the simulation, 3 scenarios are realized (Table 5). Based on the current situation, the Scenario 1 serves as a starting point for the development of the following scenarios. In the Scenario 2, the same passenger flow is preserved, but the waiting line area is increased before boarding passes are checked. In the Scenario 3, an increase in the passengers' flow by $30 \%$ (according to the forecast for 2023) is realized and measures are taken to increase the throughput of the security control area by changing the layout of the premises and equipment of 2 new passenger service lines. 


\subsection{Simulation results}

The developed models have been verified through animation-based and protocol-based tracing. The numerical simulation results were also compared with the available statistical data. As a result, the developed models have been approved as credible. After that, models of the terminal have been applied to evaluate 3 scenarios.
Figure 11 shows an unacceptably long line of passengers that forms in the departure lounge and becomes the obstacle for their movement. Figure 12 shows that this part of the queue was successfully placed in the new waiting line area. During high traffic hours, passengers pass through 2 lines: before checking boarding passes and before checking security. The bottleneck that determines the throughput of the entire system is the security check zone.

Table 5. Scenarios for analysis

\begin{tabular}{|l|c|c|c|}
\hline \multicolumn{1}{|c|}{ Characteristic } & Scenario 1 & Scenario 2 & Scenario 3 \\
\hline Infrastructurea & current & improved & improved \\
\hline Passenger flow & current & current & 47 \\
\hline Number of flight departures & 36 & 36 & $8: 00 \ldots 15: 00$ \\
\hline Simulation time & $8: 00 \ldots 15: 00$ & $8: 00 \ldots 15: 00$ & $8: 55 \ldots 14: 55$ \\
\hline Departure time & $8: 55 \ldots 14: 55$ & $8: 55 \ldots 14: 55$ & 47 \\
\hline Number of flight departures & 36 & 36 & 5901 \\
\hline Passenger number & 3701 & 3701 & 11 \\
\hline Security lines & 9 & 9 & \\
\hline
\end{tabular}

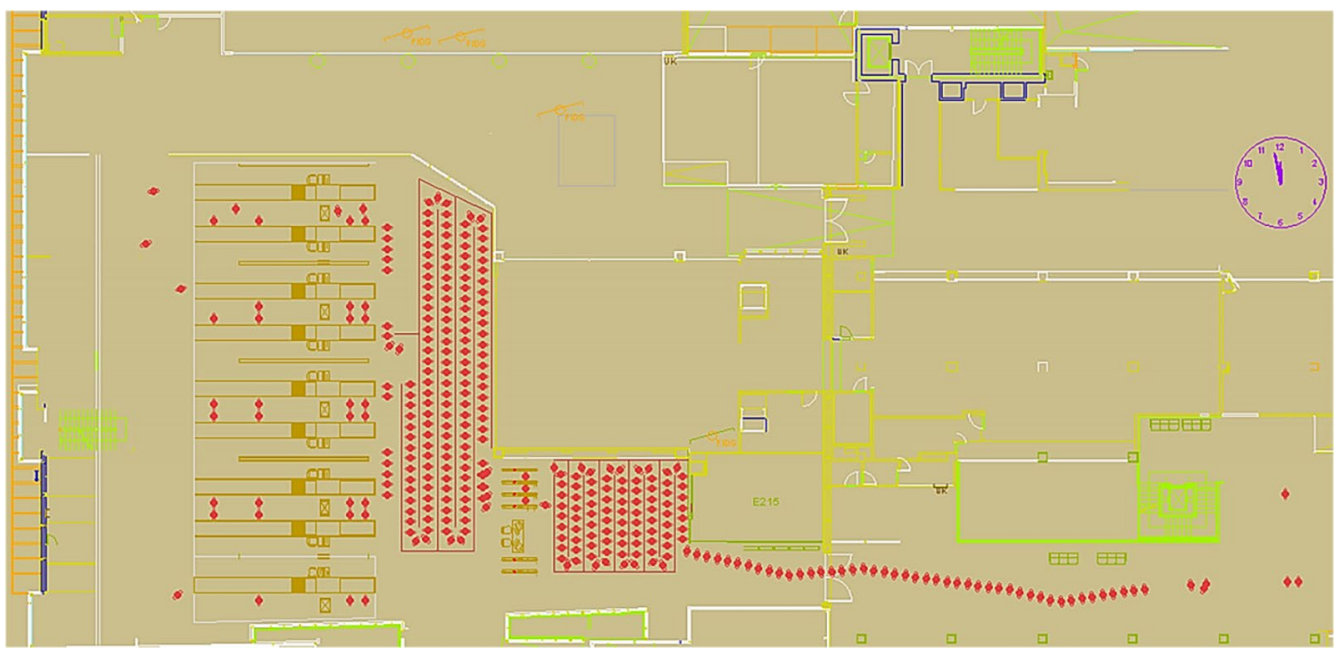

Figure 11. Visualisation of results for Scenario 1

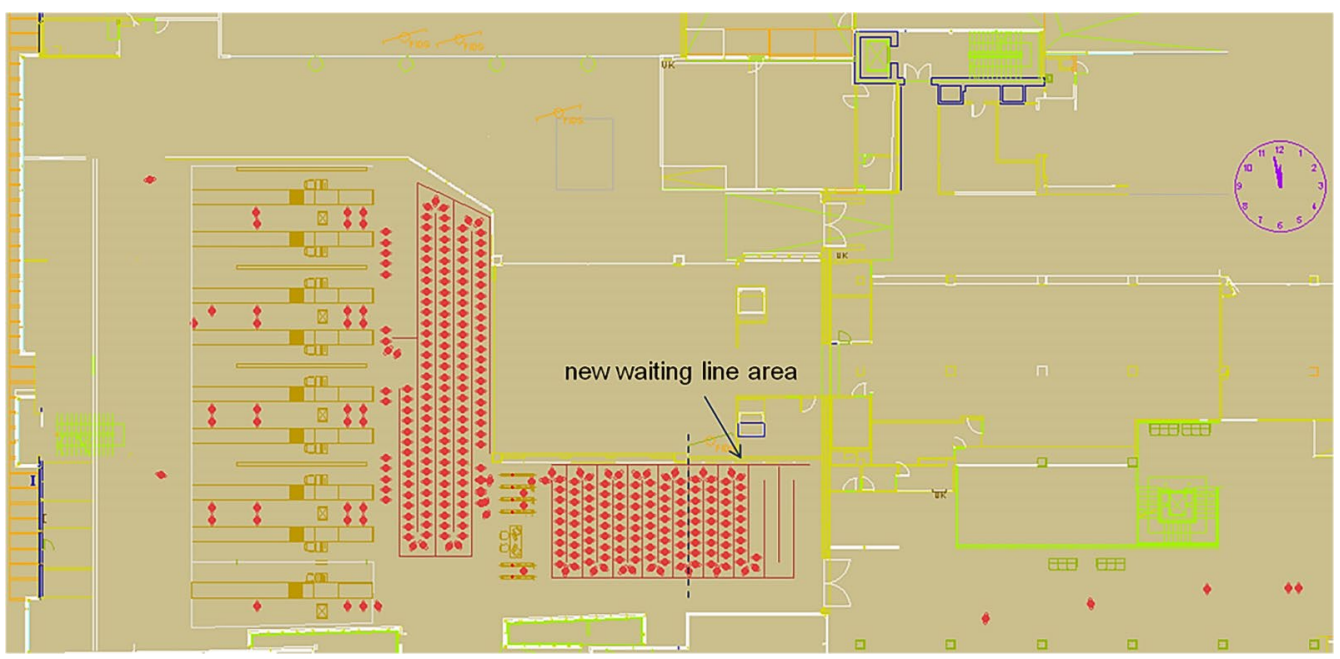

Figure 12. Visualisation of results for Scenario 2 
The average total time spent by passengers in both queues for Scenarios 1 and 2 is the same, since the geometry of the queue does not affect the waiting time. During a high intensity of passenger flows around 12:00, this time was $(23.8 \pm 2) \mathrm{min}$. Figure 13 shows the situation at 12:00, which occurs when simulating Scenario 3. Although the capacity of the system was increased, due to the high intensity of the passenger flow, the time spent in both queues increased to $(38.3 \pm 2) \mathrm{min}$. For example, in Figure 14, you can see that a queue of 73 passengers has formed in the check-in area of the AirBaltic (https://www.airbaltic.com) carrier. This situation has developed under the condition that the airline uses 3 check-in channels, and the average service time for one passenger is $89 \mathrm{~s}$.

The pivotal research question was - "will the capacity of the terminal be sufficient for the expected passenger flows?". The developed models make it possible to evaluate various specific indicators of the processes of servicing departing passengers. These indicators include, for instance, the expected time spent by a particular passenger in each of the queues and the total time of the stay in the airport premises. Special statistics are related to queuing process- es. The models provide data on personnel's workload at all workplaces involved in the passenger service process. Based on the results of the simulation, several bottlenecks and system vulnerabilities have been identified. Besides, several questions that require special decisions by the airport management were formulated. Such decisions were made, as a result of which the stability and safety of the processes of servicing the flows of departing passengers was achieved.

\section{Conclusions}

Different kinds of scenarios and decisions can be analysed based on simulation models. The use of simulation should refer to the tactical and strategic level of decision-making. The simulation requires significant efforts and results depend much on input data availability, but the advantages of the simulation allow more sustainable decisions for a system as complex as the airport.

The advantages of simulation include:

") can be used to study complex systems that would otherwise be difficult to investigate;

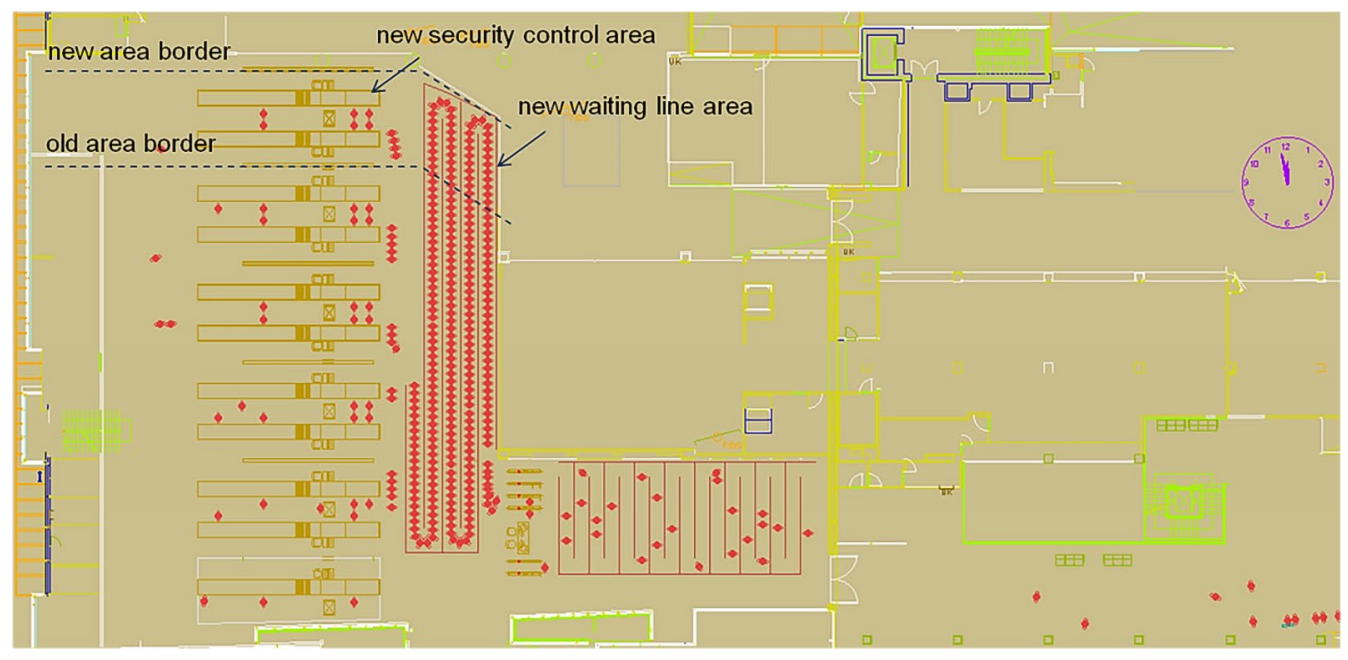

Figure 13. Visualisation of results for Scenario 3

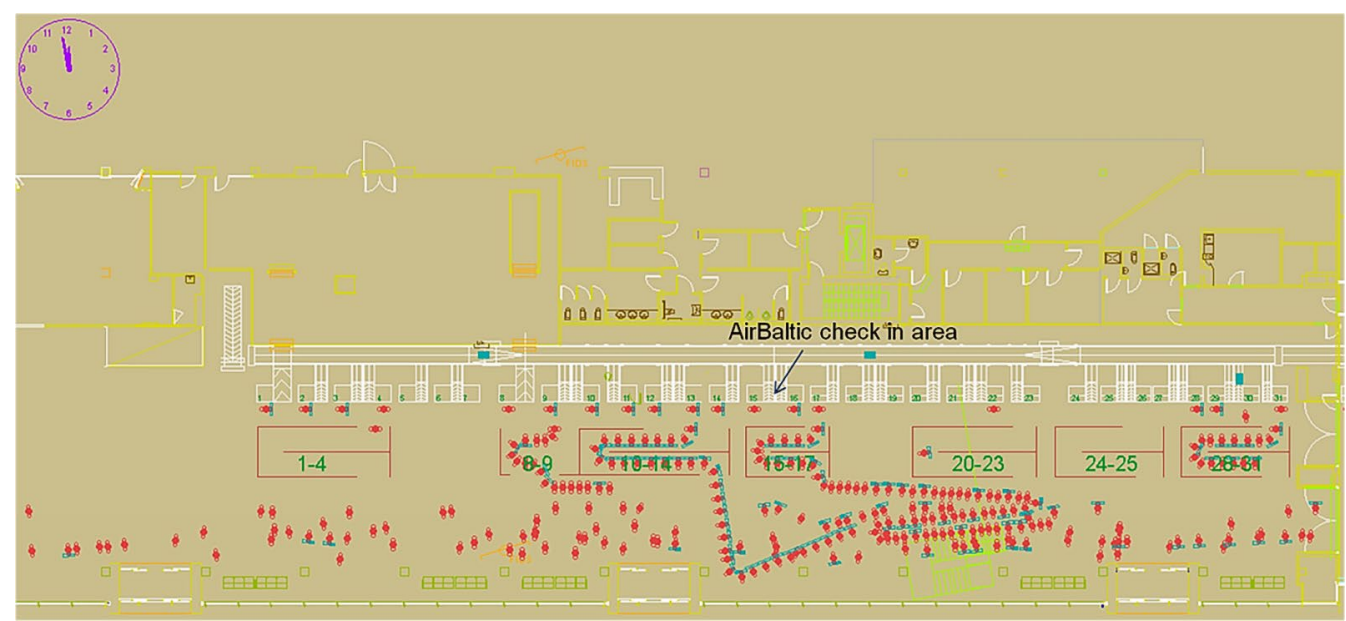

Figure 14. Visualisation of the check-in area for Scenario 3 
"») can be used to investigate situations that would be dangerous in real life;

"» ability to obtain quantitative data about the object of investigation.

The planned massive development of RIX and the surrounding area requires detailed analysis for increasing its positive impact on regional and national business economics, social aspects, and the environment. Both cases presented in the frame of the current publication serve the needs of the airport authorities to make the decisions on strategic and tactical level about the development scenarios of the airport infrastructure.

The 1st case targeted on evaluation of the surrounding transport infrastructure. Case presents the results of the evaluation of 4 development scenarios, which refer to the different stages of the infrastructure development. Developed model in frame of this case provides numerical results, which are used to evaluate the LoS for specific crossroads and evaluates the parking area utilization level. Obtained results ensure that for all crossroads the acceptable and recommended LoS has been reached. While sensitivity analysis results ensure, that crossroads have additional capacity and will be able to serve up to $10 \%$ more of traffic flow. The evaluated parking area utilization level for different development scenarios confirms the correct planning of the parking area volume guarantees additional capacity for future development.

The 2 nd case is targeted more on the evaluation of the internal infrastructure of the airport. In this case, simulation of passengers' flows has been done to evaluate the capacity and utilization level of the infrastructure elements, considering different layouts of the security lanes. 3 presented scenarios in the frame of the case consider different layouts and growth of the passenger volume. In this case, special attention has been put not only on obtaining numerical results but also on developing the visualization of the processes. The visualization, in this case, is used to demonstrate the impact of different scenarios on the passenger's queue. Obtained results demonstrate the importance of updating the airport infrastructure. This one is confirmed by the significant growth of the passenger processing time in the frame of the third scenario.

Simulation results are vivid for further economic analysis, for example, CBA (Friedrich et al. 2016). Simulation allows to study complex systems and evaluate global tendencies (considering direct and indirect impacts).

\section{Author contributions}

All authors are members of SimLab at the Transport and Telecommunication Institute (Riga, Latvia) and conceived of the presented idea of paper.

Mihails Savrasovs, Irina Yatskiv (Jackiva) and Ilya Jackson were contributors to project realization described in the 1st case study; Irina Yatskiv (Jackiva), Jurijs Tolujevs and Ilya Jackson executed the 2nd case study.
Mihails Savrasovs and Irina Yatskiv (Jackiva) were responsible for paper preparation.

All authors reviewed the results and approved the final version of the manuscript.

\section{Disclosure statement}

Authors have no competing financial, professional, and personal interests from other parties.

\section{References}

Alodhaibi, S.; Burdett, R. L.; Yarlagadda, P. K. D. V. 2020. An analytical optimisation framework for airport terminal capacity expansion, Complexity 2020: 2976281.

https://doi.org/10.1155/2020/2976281

ARC. 2021. CAST Software \& Solutions and Worldwide Aviation Consulting. Airport Research Center (ARC), Aachen, Germany. Available from Internet: https://arc.de

ATAC. 2018. Simmod PRO!. ATAC Corporation, Santa Clara, CA, US. Available from Internet: https://www.atac.com/simmod-pro/

BRG. 2017. Latvia: an Essential Global Strategic Transit Hub. Brussels Research Group (BRG), Brussels, Belgium. Available from Internet: https://brusselsresearchgroup.org/thetransport-transit-and-storage-sectors-contributed-roughly9-to-latvias-gdp/

Cascetta, E.; Cartenì, A.; Pagliara, F.; Montanino, M. 2015. A new look at planning and designing transportation systems: a decision-making model based on cognitive rationality, stakeholder engagement and quantitative methods, Transport Policy 38: 27-39. https://doi.org/10.1016/j.tranpol.2014.11.005

Chen, Y.; Wu, C.-L.; Lau, P. L.; Tang, N. Y. A.; Ma N. K.; Chung, Y.-S. 2019. Airport passenger shopping modeling and simulation: targeting distance impacts, in 2019 Winter Simulation Conference (WSC), 8-11 December 2019, National Harbor, MD, US, 524-535. https://doi.org/10.1109/ WSC40007.2019.9004776

CLOSER. 2012. Deliverable D4.2: Policy Advisory Group Recommendations. Connecting LOng and Short-distance networks for Efficient tRansport (CLOSER) Project. 46 p. Available from Internet: https://trimis.ec.europa.eu/sites/default/files/project/documents/20130513_124334_59698_deliverabled42.pdf

Friedrich, M.; Leurent, F.; Jackiva, I.; Fini V.; Raveau, S. 2016. From transit systems to models: purpose of modelling, in G. Gentile, K. Noekel (Eds.). Modelling Public Transport Passenger Flows in the Era of Intelligent Transport Systems. https://doi.org/10.1007/978-3-319-25082-3_4

Ginzberg, M. J.; Stohr, E. A. 1982. Decision Support Systems: Issues and Perspectives. NYU Working Paper No. IS-82-12. 42 p. Available from Internet: https://ssrn.com/abstract $=1290170$

Gök, Y. S.; Tomasella, M.; Guimarans, D.; Ozturk, C. 2020. A simheuristic approach for robust scheduling of airport turnaround teams, in 2020 Winter Simulation Conference (WSC), 14-18 December 2020, Orlando, FL, US, 1336-1347. https:// doi.org/10.1109/WSC48552.2020.9383947

Jeppesen. 2021. Jeppesen Total Airspace and Airport Modeler. Jeppesen - a Boeing Company, US. Available from Internet: https://ww2.jeppesen.com/airspace-solutions/total-airspaceand-airport-modeler/ 
Jeppesen. 2015. Total Airspace and Airport Modeller (TAAM): Product Profile. Jeppesen - a Boeing Company, US. 13 p. Available from Internet: http://ww1.jeppesen.com/documents/aviation/government/TAAM-product-profile.pdf

López, E. C.; Marmier, F.; Fontanili, F. 2019. Bus fleet size dimensioning in an international airport using discrete event simulation, in 2019 Winter Simulation Conference (WSC), 8-11 December 2019, National Harbor, MD, US, 464-475. https://doi.org/10.1109/WSC40007.2019.9004878

MoT. 2006. Transport Development Guidelines 2007-2013. Ministry of Transport (MoT) of the Republic of Latvia.

Ortúzar, J. D.; Willumsen, L. G. 2011. Modelling Transport. John Wiley \& Sons, Ltd. 608 p. https://doi.org/10.1002/9781119993308

PTV. 2005. VISSIM 4.10: User Manual. PTV Planung Transport Verkehr AG, Karlsruhe, Germany. 310 p.

RIX. 2021. About RIX. Riga International Airport (RIX), Latvia. Available from Internet: https://www.riga-airport.com/aboutcompany/en

Saifutdinov, F.; Jackson, I.; Tolujevs, J.; Zmanovska, T. 2020. Digital twin as a decision support tool for airport traffic control, in 2020 61st International Scientific Conference on Information Technology and Management Science of Riga Technical University (ITMS), 15-16 October 2020, Riga, Latvia, 1-5. https://doi.org/10.1109/ITMS51158.2020.9259294

San Antonio, A.; Juan, A. A.; Calvet, L.; Fonseca i Casas, P.; Guimarans, D. 2017. Using simulation to estimate critical paths and survival functions in aircraft turnaround processes, in 2017 Winter Simulation Conference (WSC), 3-6 December 2017, Las Vegas, NV, US, 3394-3403. https://doi.org/10.1109/WSC.2017.8248055

Scala, P.; Mujica, M.; Delahaye, D.; Ma, J. 2019. A generic framework for modeling airport operations at a macroscopic level, in 2019 Winter Simulation Conference (WSC), 8-11 December 2019, National Harbor, MD, US, 512-523. https://doi.org/10.1109/WSC40007.2019.9004865

Scala, P.; Mujica, M.; Wu, C.-L.; Delahaye, D. 2018. December. Sim-Opt in the loop: algorithmic framework for solving airport capacity problems, in 2018 Winter Simulation Conference (WSC), 9-12 December 2018, Gothenburg, Sweden, 22612272. https://doi.org/10.1109/WSC.2018.8632531

Schultz, M. 2017. Faster aircraft boarding enabled by infrastructural changes, in 2017 Winter Simulation Conference (WSC), 3-6 December 2017, Las Vegas, NV, US, 2530-2541. https://doi.org/10.1109/WSC.2017.8247981

Simio LLC. 2016. Airport Simulation Software. Simio LLC, Sewickley, PA, US. Available from Internet: https://www.simio. com/applications/airport-simulation-software/

Tomasella, M.; Clare, A.; Gök, Y. S.; Guimarans, D.; Ozturk, C. 2019. STTAR: a simheuristics-enabled scheme for multistakeholder coordination of aircraft turnaround operations, in 2019 Winter Simulation Conference (WSC), 8-11 December 2019, National Harbor, MD, US, 488-499. https://doi.org/10.1109/WSC40007.2019.9004787

Tomasella, M.; Hancock, P.; Hristova, B.; Vancova, Z.; Buke, B. 2017. Enhancing the toolkit of airport operations analysts: evidence from an airport baggage handling system improvement project, in 2017 Winter Simulation Conference (WSC), 3-6 December 2017, Las Vegas, NV, US, 2542-2553. https://doi.org/10.1109/WSC.2017.8247982

TTI. 2021. SimLab - Laboratory of Applied Modelling. Transport and Telecommunication Institute (TTI), Riga, Latvia. Available from Internet: https://tsi.lv/research/research-at-tsi/ research-support-structure/simlab-laboratory-of-appliedmodelling/

Vitor, F.; Santos, V. A.; Chwif, L. 2016. Warnings about simulation revisited: improving operations in Congonhas airport, in 2016 Winter Simulation Conference (WSC), 11-14 December 2016, Washington, DC, US, 2418-2429.

https://doi.org/10.1109/WSC.2016.7822281

Yatskiv (Jackiva), I.; Savrasovs, M.; Gromule, V.; Zemljanikins, V. 2016. Passenger terminal safety: simulation modelling as decision support tool, Procedia Engineering 134: 459-468. https://doi.org/10.1016/j.proeng.2016.01.068

Yurshevich, E. 2013. Methodology of Decision-Making Support Based on Urban Transportation System Microscopic Models Repositories: Doctoral Thesis. Transport and Telecommunication Institute, Riga, Latvia. 204 p. 